

$$
\begin{aligned}
\therefore C_{1}+\alpha \frac{\partial \varphi}{\partial t} & =-\frac{\gamma M}{\mu_{0}} \sin \varphi \cos \varphi \\
\frac{\partial \varphi}{\partial t}-\alpha C_{1} & =\gamma H_{z} \\
0 & =\frac{M}{\mu_{0}} \sin ^{2} \varphi+H_{k}-C_{2}{ }^{2} C_{A}
\end{aligned}
$$

in which $\mathrm{H}_{\mathrm{K}}=2 \mathrm{~K} / \mathrm{M}, \mathrm{C}_{\mathrm{A}}=2 \mathrm{~A} / \mathrm{M}$, and $\mathrm{H}_{2}$ is the applied field.

\section{Approximate Solution}

An approximate solution is obtained if the additional assumption is made that $\varphi$, the angle associated with the component of $M$ perpendicular to the wall plane, is smal1. From eqs. $2 a$ and $2 b$ with $\sin \varphi \cong \varphi$ and $\cos \varphi \cong 1$, a linear differential equation in $\varphi$ results, and the solution for a step function applied field is

$$
\varphi=\frac{\mu_{0} H z}{\alpha M}\left(1-e^{-\frac{\alpha}{1+\alpha^{2}} \frac{\gamma M}{\mu_{0}} t}\right)
$$

and

$$
c_{1}=-\alpha y H_{z}-\frac{\gamma H_{z}}{\alpha}\left(1-e^{-\frac{\alpha}{1+\alpha^{2}} \frac{\gamma M}{\mu_{0}} t}\right) \text {. }
$$

From eq. $2 \mathrm{c}, \mathrm{C}_{2}$ is obtained as

$$
c_{2}=\left[\frac { \mu _ { 0 } H ^ { 2 } } { \alpha ^ { 2 } M } \left(1-e^{\left.\left.-\frac{\alpha}{1+\alpha^{2}} \frac{M}{\mu_{0}}\right)^{2}+H_{k}\right]^{\frac{1}{2}} / c_{A}^{\frac{1}{2}}}\right.\right.
$$

Finally, the velocity is obtained from $v=d y / d t=-(\partial \theta / \partial t) /(\partial \theta / \partial y)=-c_{1} / c_{2}$ as

$$
\begin{aligned}
& v=\frac{\gamma H_{z}}{\alpha}(A / K)^{\frac{1}{2}}\left(1-e^{-\alpha \frac{\gamma M}{\mu_{0}} t}\right)\left[1+\frac{h_{z}^{2}}{\alpha^{2} h_{k}}\left(1-e^{-\alpha \frac{\gamma M}{\mu_{0}} t}\right)^{2}\right]^{-\frac{1}{2}}, \\
& \text { with } h_{z}=\mu_{0} H_{z} / M, \text { and } h_{k}=\mu_{0} H_{k} / M, \\
& \text { and } \alpha^{2}<<1 .
\end{aligned}
$$

The steady-state velocity corresponds to the solution previously obtained by Feldtkeller [1]. The effective time constant for $\alpha=0.01$ and $M=1.0 \mathrm{w} / \mathrm{m}^{2}$ is 0.5 nanosecond. The transient behavior of: $C_{2}:$ corresponds to the wall 
contraction that takes place as the wall accelerates. $C_{2}$ may also be displäyed as a function of velocity.

$$
C_{2}=\left[A / K-\mu_{0} v^{2} / 2 \gamma^{2} K\right]^{\frac{1}{2}} \text {. }
$$

For small $v$ or $\operatorname{small} \mathrm{H}_{z}$, the wall contraction is negligible and the velocity increases to the final value as a simple exponential.

\section{"Exact" Solution}

Eqs. 2 may also be integrated directly to yield:

$$
\sin ^{2} \varphi=\frac{A B\left(1-e^{-\beta t}\right)^{2}}{\left(A-B e^{-\beta t}\right)^{2}+A B\left(1-e^{-\beta t}\right)^{2}}
$$

and

in which

$$
\sin \varphi \cos \varphi=\frac{(A B)^{\frac{1}{2}}\left(1-e^{-\beta t}\right)\left(A-B e^{-\beta t}\right)}{\left(A-B e^{-\beta t}\right)^{2}+A B\left(1-e^{-\beta t}\right)^{2}},
$$

$$
\begin{aligned}
& A=1+\left[1-\left(\frac{2 h z}{\alpha}\right)^{2}\right]^{\frac{1}{2}}, \beta=\frac{\alpha}{1+\alpha^{2}}\left[1-\left(\frac{2 h}{\alpha}\right)^{2}\right]^{\frac{1}{2}} \frac{\gamma M}{\mu_{0}}, \\
& B=1-\left[1-\left(\frac{2 h z}{\alpha}\right)^{2}\right]^{\frac{1}{2}}, h_{z}=\mu_{0} H_{z} / M: .
\end{aligned}
$$

The time dependence of the velocity constant, the structure constant, and the velocity are given by

$$
\begin{aligned}
& C_{1}=\frac{1}{1+\alpha^{2}}\left[-\alpha \gamma H_{2}-\frac{\gamma M}{\mu_{0}} \sin \varphi \cos \varphi\right], \\
& C_{2}=\left(\frac{M}{\mu_{0}} \sin ^{2} \varphi+H_{k}\right)^{\frac{1}{2}} C_{A}{ }^{\frac{1}{2}}, \\
& v=-C_{1} / C_{2} .
\end{aligned}
$$

For $2 h_{z} / \alpha<<1$ but not $\left(h_{z} / \alpha\right)^{2}<<h_{k}$ with $h_{k}=\mu_{o} H_{k} / M$; the solution reduces to the previous approximate solution. The steady-state velocity is given by

$$
\mathrm{v}=\frac{\gamma_{\mathrm{H}} \mathrm{z}}{\alpha}(\mathrm{A} / \mathrm{K})^{\frac{1}{2}}\left[1+\frac{1}{2 \mathrm{~h}_{\mathrm{k}}}\left(1-\sqrt{1}-\left(\frac{2 \mathrm{~h}_{\mathrm{z}}}{\alpha}\right)^{2}\right)\right]^{-\frac{1}{2}}
$$

which is the Walker solution [2] discussed in detail by Schlömann [3], [4] The solution is 1 imited by $2 \mathrm{~h}_{z} / \alpha=1$ corresponding to. $\varphi=45^{\circ}$ and

$$
\left.v\right|_{h_{z}=\alpha / 2}=\gamma\left(\frac{2 A}{\mu_{0}}\right)^{\frac{1}{2}}\left(2+4 h_{k}\right)^{-\frac{1}{2}} \text {. }
$$


Note that the effective damping constant, $\beta$, in the transient solution is zero at this point although the overall time response remains finite. The peak velocity and the corresponding applied field are given by

$$
\begin{aligned}
& \mathrm{v}_{\mathrm{pk}}=\gamma\left(\frac{2 \mathrm{~A}}{\mu_{\mathrm{o}}}\right)^{\frac{1}{2}}\left[\left(1+\mathrm{h}_{\mathrm{k}}\right)^{\frac{1}{2}}-\mathrm{h}_{\mathrm{k}}^{\frac{1}{2}}\right], \\
& \left.\mathrm{h}_{\mathrm{z}}\right|_{\mathrm{v}}=\alpha \mathrm{h}_{\mathrm{kk}}^{\frac{1}{4}}\left(1+\mathrm{h}_{\mathrm{k}}\right)^{\frac{1}{4}}\left[\left(1+\mathrm{h}_{\mathrm{k}}\right)^{\frac{1}{2}}-\mathrm{h}_{\mathrm{k}}^{\frac{1}{2}}\right] .
\end{aligned}
$$

The motion predicted by this "exact" solution for a planar wall is wellbehaved for $2 h_{z} / \alpha \leq 1$. Schlömann [4] points out that there may be reason to doubt that the solution is valid for fields larger than $\left.h_{z}\right|_{v}=v_{p k}$ Slonczewski [5] reasons that if the wall is described by a negative differential mobility then instabilities characterizerized by corrugated variations along the wall may develop and the assumption of a planar wall is violated. The "exact" transient solution predicts that velocity changes in the negative differential mobility region are characterized by long time constants possibly requiring that the instabilities develop slowly. Experimental evidence in permalloy films indicates that a corrugated structure is much more likely to appear with relatively long pulses and that the wall remains planar for relatively short pulses.

A family of curves of the steady-state solution is shown in Fig. 2 for various $h_{k}$ and normalized drive field, $2 h_{z} / \alpha$. A corresponding transient response for a particular $h_{k}$ and various drives is shown in Fig. 3. Notice that overshoots in the velocity are predicted for small $h_{k}$ and large drives. Summary

Analytic solutions are found for transient domain wall velocity in bulk uniaxial ferromagnetic materials excited by a step function easy-axis field. The solutions reduce to previously obtained steady-state solutions. The response time(s) associated with wall contraction and velocity is of the order of nanoseconds for permalloy materials but may be much longer for ortho-ferrite materials. 
References

1. E. Feldtkeller, "Magnetic Domain Wall Dynamics", Phys. Stat. Sol. 27, pp 161-170, 1968 .

2. I. R. Walker quoted by J. F. Dillon, "Magnetism", vol. III edited by G. Rado and H. SuhI, Academic Press, pp 450-453, 1963.

3. E. Schlömann, Appl. Phys. Letters 19, 274, 1971.

4. E. Schlömann, Paper 3E-7, Magnetism Conference, Chicago, Nov. 1971.

5. J. C. Slonczewski, "Dynamics of Magnetic Domain Walls", IBM Report RC 3534, Watson Research Center, Yorktown Heights, N.Y., Sept. 1971. 


\section{Figure Captions}

Fig. 1. Coordinate system.

Fig. 2. Normalized velocity versus normalized applied field from the exact steady-state solution.

Fig. 3. Normalized velocity versus normalized time from the exact theory with normalized drive as a parameter. 


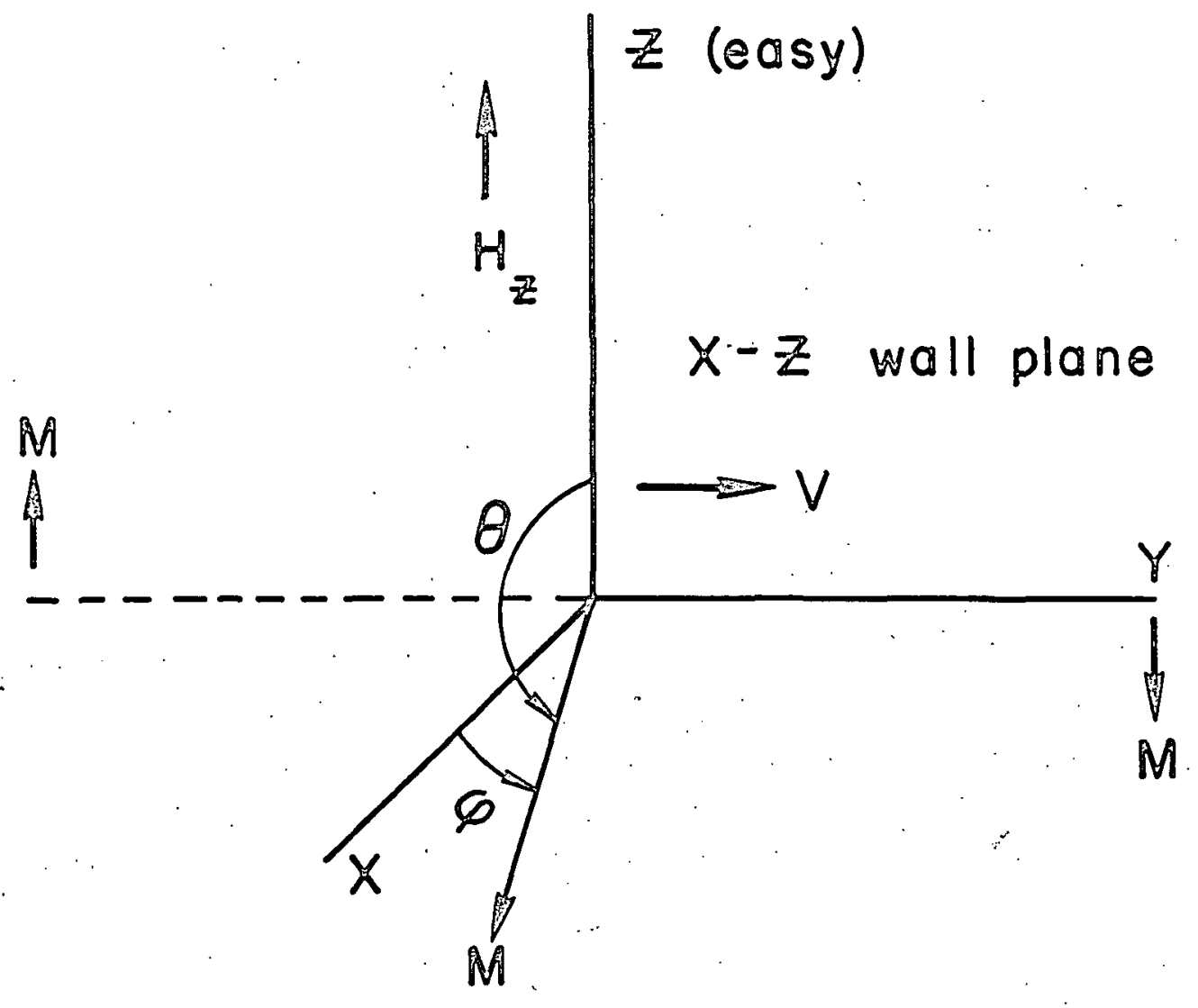

Fir i

Bourne Bistram 


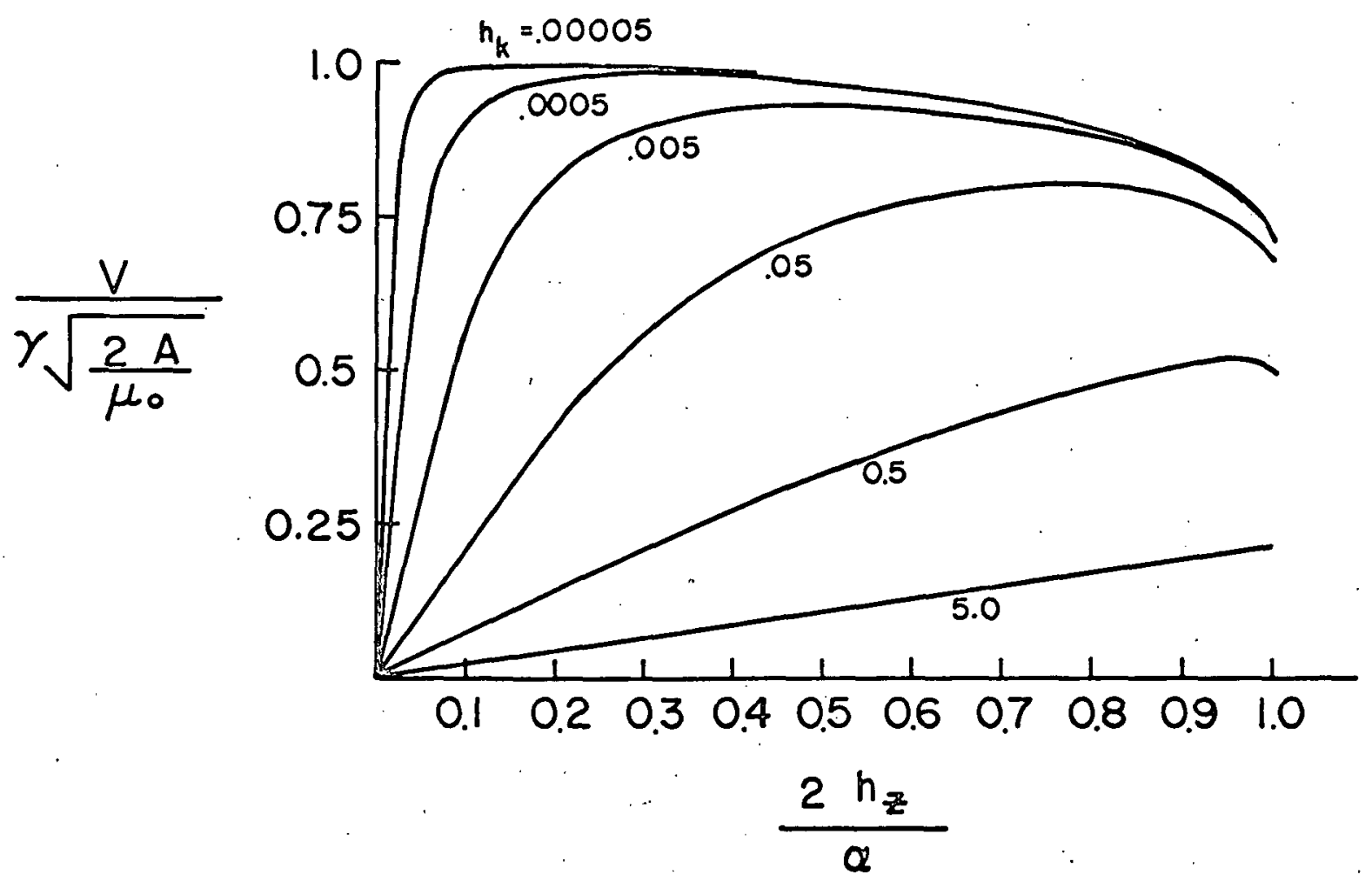

Fir, 2

Bournez Butrom 

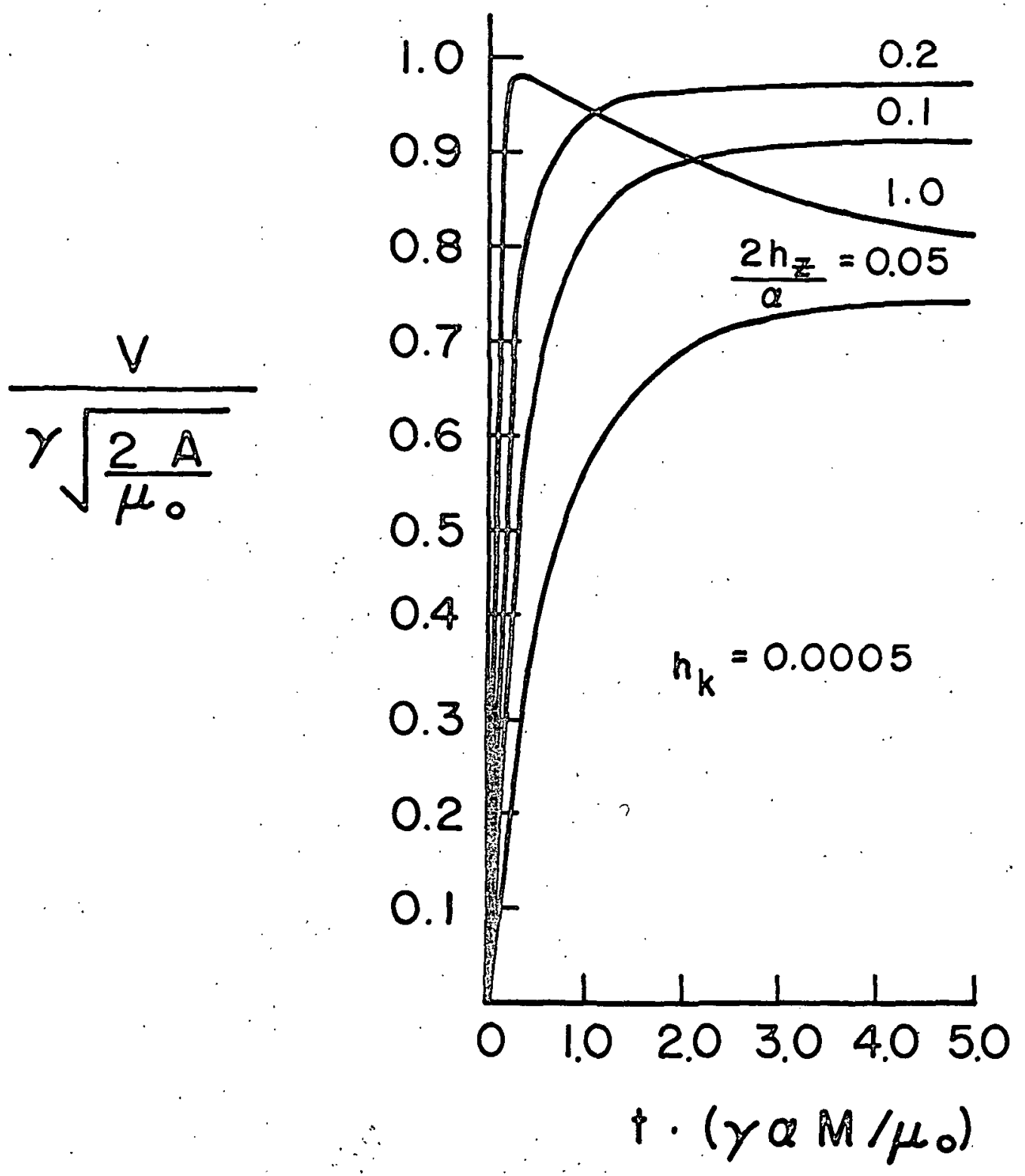